\title{
MicroRNA-203 contributes to skin re-epithelialization
}

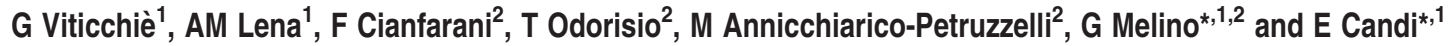

Keratinocyte proliferation and migration are crucial steps for the rapid closure of the epidermis during wound healing, but the molecular mechanisms involved in this cellular response remain to be completely elucidated. Here, by in situ hybridization we characterize the expression pattern of miR-203 after the induction of wound in mouse epidermis, showing that its expression is downregulated in the highly proliferating keratinocytes of the 'migrating tongue', whereas it is strongly expressed in the differentiating cells of the skin outside the wound. Furthermore, subcutaneous injections of antagomiR-203 in new born mice dorsal skin strengthened, in vivo, the inverse correlation between miR-203 expression and two new target mRNAs: RAN and RAPH1. Our data suggest that miR-203, by controlling the expression of target proteins that are responsible for both keratinocyte proliferation and migration, exerts a specific role in wound re-epithelialization and epidermal homeostasis re-establishment of injured skin.

Cell Death and Disease (2012) 3, e435; doi:10.1038/cddis.2012.174; published online 29 November 2012

Subject Category: Experimental Medicine

The repair of damaged skin is a highly dynamic and complex process involving three partially overlapping main phases: the early inflammatory phase, the intermediate proliferative phase, which includes re-epithelialization, angiogenesis and granulation tissue formation, and finally the late tissue remodeling phase. ${ }^{1}$ After injury, keratinocytes at the wound edges revert their terminal differentiation in a process called 'keratinocyte activation', during which they acquire migratory potential and facilitate wound closure. ${ }^{2-5}$ The capacity to heal wounds relies on the rapid proliferation and migration of epithelial cells located at the wound margin and their ability, once the wound bed has been completely covered by an epidermal sheath, to reset the terminal differentiation program, restoring a complete stratified epithelium. ${ }^{1,6,7}$ The molecular mechanisms involved in wound re-epithelialization are still not fully elucidated. Different models emphasize the role of skin stem cells, of basal and suprabasal keratinocytes at the wound edges or of hair follicles of the wound surrounding areas, ${ }^{8-10}$ and it is evident that during reepithelialization epidermal layers undergo different morphological changes, which include loss of intercellular adhesions, cellular polarity and cytoskeleton reorganization to restore local homeostasis and epidermal barrier function. ${ }^{11,12}$ The events described during cutaneous wound healing are due to a fine modulation of gene expression and involve different signaling pathways. ${ }^{13-15}$ In this scenario, microRNAs (miRNA) could play a fundamental role. ${ }^{16}$ MiRNAs, conserved small non-coding RNAs (19-22 nucleotides in length), usually bind the $3^{\prime}$-untranslated region $\left(3^{\prime}\right.$-UTR) of the target messenger RNAs (mRNAs) and are able to inhibit their translation or to induce their degradation. ${ }^{17,18}$ MiRNAs have recently been shown to play pivotal roles in skin development and pathologies regulating a lot of different cellular processes, such as proliferation, differentiation, death and transformation. ${ }^{19-24}$ MiR-203 is the most abundant keratinocyte-specific miRNA in the epidermis. ${ }^{25}$ It has an antiproliferative role, fundamental for skin development and differentiation, targeting the $3^{\prime}$-UTR of the transcription factor p63. ${ }^{22,26}$ Indeed, p63, a member of the p53 family, has an important role in maintaining basal keratinocyte proliferative potential in adult normal epidermis, ${ }^{27-33}$ during development ${ }^{34-37}$ and in pathological conditions. ${ }^{23,24,38-41}$ MiR-203 downregulation, as described in several cancers, ${ }^{42-46}$ promotes EMT transition and enables tumor cells to acquire invasive metastatic features. ${ }^{45,47}$ To further investigate miR-203 function in pathological conditions, we decided to study its possible role in the re-epithelialization process during wound healing. We found that miR-203 expression is downregulated in the leading edge of the migrating tongue. Moreover, we identified RAN and RAPH1 as new miR-203 targets and evaluated their role in cell proliferation and migration during wound repair together with p63 and LASP1, two other known miR-203 targets. Our findings indicate that miR-203 expression is highly controlled in the wound area and that miR-203 could actively contribute to cutaneous wound re-epithelialization.

\section{Results}

Characterization of miR-203 expression during wound healing. To analyze miR-203 expression in injured skin,

\footnotetext{
'Department of Experimental Medicine and Surgery, University of 'Tor Vergata', Via Montpellier, 1, Rome 00133, Italy and 'Istituto Dermopatico dell'Immacolata-Istituto di Ricovero e Cura a Carattere Scientifico (IDI-IRCCS), Via Monti di Creta, 104, Rome 00166, Italy

${ }^{\star}$ Corresponding author: G Melino or E Candi, Department of Experimental Medicine and Surgery, University of Tor Vergata, Via Montpellier, 1, Rome 00133, Italy. Tel: + 39067259 6976; Fax: + 39067259 6977; E-mail: gerry.melino@uniroma2.it (GM) or Tel: + 39067259 6487; Fax: + 3906 7259 6977; E-mail: candi@uniroma2.it (EC)

Keywords: microRNA; wound healing; p63; keratinocytes

Abbreviations: miRNA, microRNA; HEKn, human epidermal keratinocytes neonatal; BrdU, 5-bromo-2'-deoxyuridine; PI, propidium iodide; K6, keratin 6; H\&E, hematoxylin and eosin staining

Received 08.8.12; revised 27.9.12; accepted 05.10.12; Edited by RA Knight
} 

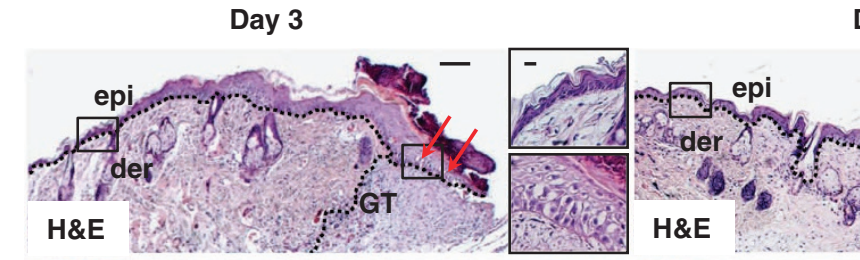

Day 5
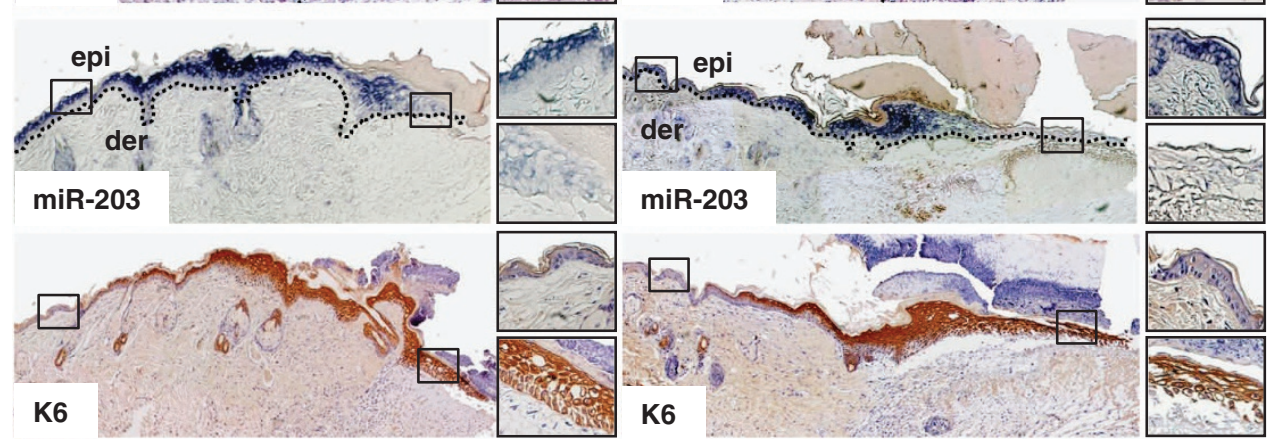

Figure 1 MiR-203 expression in mouse skin after wound infliction. Histological analysis of the wounded mouse epidermis at days 3 and 5 after wounding. From the top: $\mathrm{H} \& \mathrm{E}$, in situ hybridization performed using a specific miR-203 probe, IHC analysis of $\mathrm{K} 6$. Black bars: $100 \mu \mathrm{m}$. Each panel is a composition of six images taken with an $\times 10$ objective to provide high resolution over a wide area, whereas the insets are a magnification $(\times 20)$ of the identified squared boxes: left box, upper inset; right box, lower inset. Black bar in the insets: $20 \mu \mathrm{m}$. (epi: epidermis; der: dermis; GT: granulation tissue). In H\&E staining panels, red arrows indicate the wound edge. Negative controls for the in situ hybridization are shown in Supplementary Figure S1

in situ hybridizations were performed on sections of mouse skin samples collected at 3 and 5 days post wounding. The top panels of Figure 1 illustrate hematoxylin and eosin (H\&E) staining of wound specimens and red arrows indicate the wound edge. In situ hybridization (middle panels) shows a strong miR-203 expression in all suprabasal layers of the epidermis surrounding the wound and in the hyperplastic epidermis at the wound margin. In contrast, miR-203 is almost completely absent in keratinocytes at the migratory front, both at 3 and 5 days post injury. Finally, the lower panels illustrate keratin 6 (K6) staining to evidentiate the epithelial tongue of hyperproliferating and migrating keratinocytes; the $\mathrm{K} 6$ signal is absent in keratinocytes of the non-lesional epidermis.

Supplementary Figure $\mathrm{S} 1$ shows the in situ hybridization controls performed on serial tissue sections using a scrambled control sequence as a probe.

RAN and RAPH1 are new direct miR-203 targets. Recently, new miR-203 targets were identified, indicating an antiproliferative, antimigratory and anti-invasive role for this miRNA. ${ }^{22,26,45,46}$ Using TargetScan 5.1 prediction software, mRNA microarray profiling and Go-term analysis, we were able to identify a set of mRNAs downregulated after miR-203 overexpression. They codify for proteins involved in cellular growth and proliferation and/or in cellular organization, morphology and migration. ${ }^{46}$ As all these pathways are also involved in skin repair, we wanted to investigate if miR203 contributes to the re-epithelialization process after wound infliction.

We screened among a pool of genes that we have previously found to be downregulated following miRNA-203 overexpression in primary human keratinocytes by microarray analysis. ${ }^{46}$ Among the validated targets (Supplemental Figure S2a), we selected the ones whose expression was downregulated more than $50 \%$ in comparison to control experiments. For more than 10 targets, including p63, we were able to observe an inverse correlation with miR-203 expression at the mRNA level following calcium-induced keratinocyte differentiation (Supplementary Figure S2b). MiR-203, $\Delta$ Np63 and involucrin mRNAs were quantified at 0,1 and 3 days after calcium addition as positive controls. Western blots in Supplementary Figure S2c show that only four putative targets were downregulated at the protein level: p63 and LASP1, already known as miR-203 targets, and RAN and RAPH1, not previously described as regulated by miR-203. Therefore, we focused on RAN and RAPH1. Ran is a member of the small GTP-binding protein (G-protein) superfamily. It is associated with cell proliferation and survival, nucleocytoplasmic transport and cytoskeletal dynamics. ${ }^{48-50}$ Raph1, also known as lamellipoidin, is a regulator of actin dynamics taking part with roles in cytoskeleton remodeling complexes and in the acquisition of invasive cancer cell ability. ${ }^{51-53}$ The biological roles of Raph1 and Ran, as well as their expression in the basal layer of the epidermis, make them good candidates as regulators of the wound healing processes.

RAN and RAPH1 mRNAs show a conserved miR-203 target site in their $3^{\prime}$-UTR (Figure 2a). To demonstrate that miR-203 targets them directly, we cloned part of their $3^{\prime}-$ UTRs, containing the binding sites, in a luciferase reporter construct. Relative luciferase activity was quantified $24 \mathrm{~h}$ after co-transfection of reporter constructs with an miR-203 expression vector. MiR-203 overexpression repressed the luciferase expression controlled by the $3^{\prime}$-UTRs of RAN and RAPH1, where a significant decrease of about $25 \%$ in luciferase activity was observed. Deletions of miR-203 target sequences in the RAN and RAPH1 3 '-UTRs abolish the miRNA-mediated effect on luciferase activity (Figure 2a). Moreover, transfection of pre-miR-203 in human epidermal keratinocytes neonatal (HEKn) cells promoted a strong 

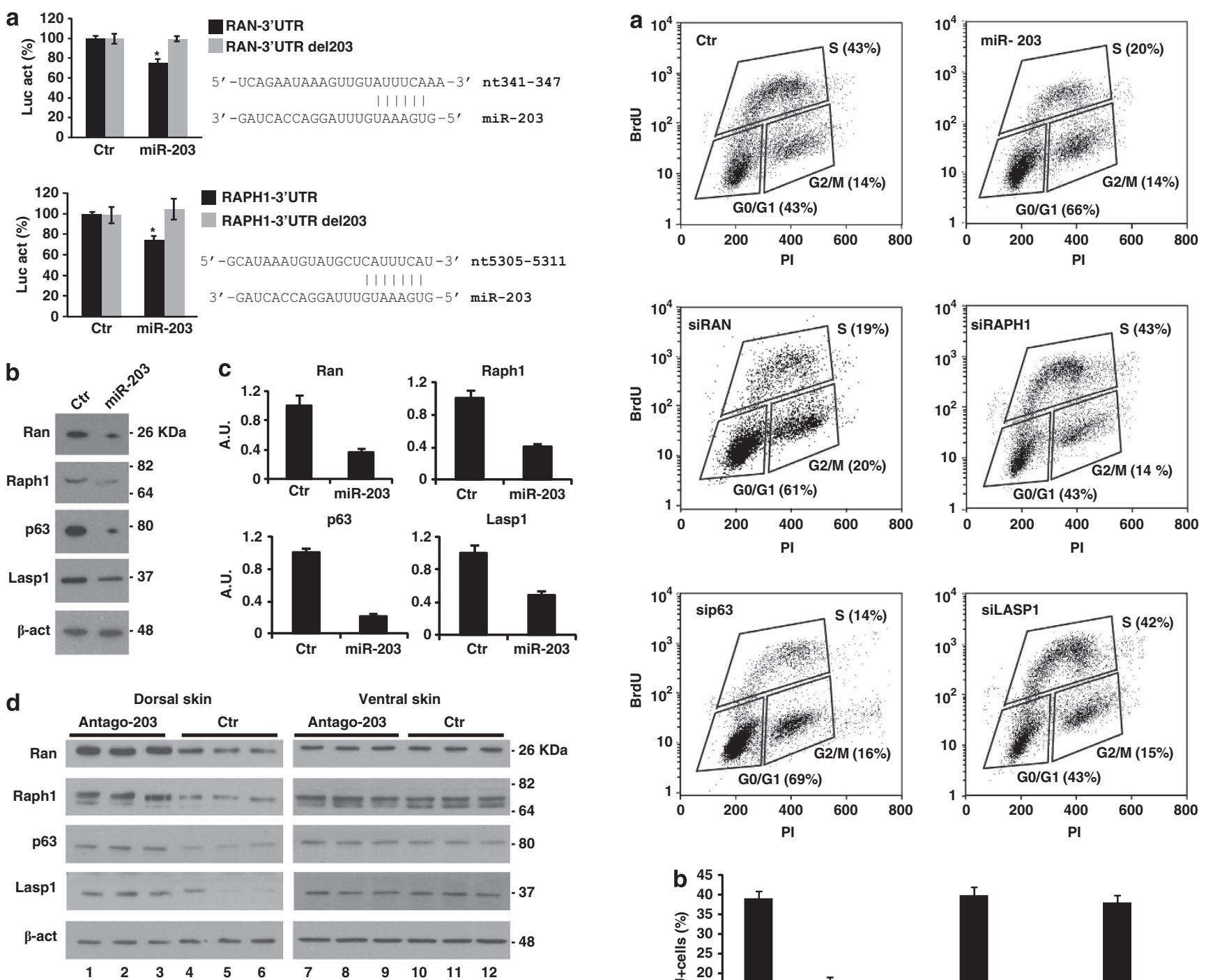

Figure 2 RAN and RAPH1 are direct miR-203 targets. (a) Insertion of miR-203 target $3^{\prime}$-UTRs in a luciferase reporter gene construct leads to a decrease in relative luciferase activity only in the presence of miR-203 target sites (black bars). Deletions of miR-203 target sequences in the RAN and RAPH1 $3^{\prime}$-UTRs abolish the miRNAmediated effect on luciferase activity (gray bars). Histograms represent the average \pm S.D. from three independent assays. ${ }^{*} P$-value $<0.01$ by Student's $t$-test. Predicted miR-203 target sites on RAN and RAPH 3'-UTRs. (b) Western blotting of miR-203 targets $48 \mathrm{~h}$ after pre-miR-203 or scrambled control sequence transfections. $\beta$-Actin expression was used as control. (c) MiR-203 target expression was normalized to actin by densitometry. Results are reported as arbitrary units (AU). (d) Western blotting of miR-203 targets in total protein extracts from dorsal (lanes 1-6) and ventral (lanes 7-12) skin biopsies of new born mice subcutaneously injected on the back side with antagomiR-203 (lanes 1-3 and 7-9) or negative control(lanes 4-6 and 10-12) sequences

reduction of Ran and Raph1 protein levels as shown in western blot analysis (Figures $2 \mathrm{~b}$ and $\mathrm{c}$ ). As shown in the densitometric analysis, the reduction obtained for these two miR-203 targets are comparable to p63 and Lasp1.

To test the in vivo effect of miR-203 on silencing its targets, subcutaneous injections of antagomiR-203 and scrambled control sequences were performed on newborn CD1 mice. ${ }^{54}$ The mean $90 \%$ reduction in miR-203 expression obtained in antagomiR-203-injected mice compared with the control

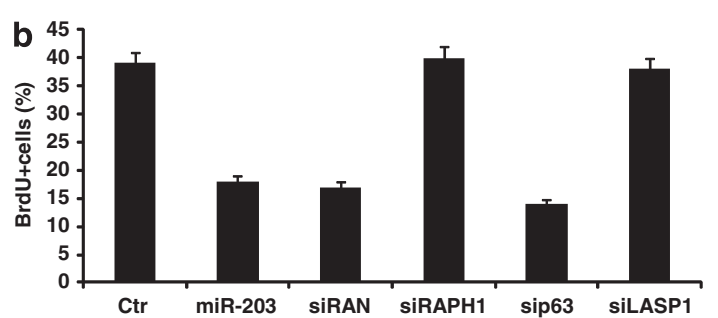

Figure 3 MiR-203 inhibits proliferation and induces G0/G1 arrest in HEKn by RAN and p63 downregulation. (a) At $48 \mathrm{~h}$ after transfection with scramble control or pre-miR-203 sequences, HEKn cells were subjected to a $4 \mathrm{~h} \mathrm{BrdU-pulse,} \mathrm{and} \mathrm{then}$ collected, PI-stained and analyzed by flow cytometry. BrdU-positive cells are indicated as the $\mathrm{S}$ phase (fluorescent populations are discriminated for PI-stained DNA content, respectively, of $2 n$ or $4 n$ fixed to values of 200 and 400 in the plots). (b) Histogram shows the relative percentage of BrdU-incorporating cells (BrdUpositive cells). Results are shown as the mean \pm S.D. from three independent experiments

group (Supplementary Figure S3b) had no consequences on skin morphology, as shown by H\&E stainings on skin sections of mice of the two experimental groups (Supplementary Figure S3a). Western blots performed on protein extracts, obtained from dorsal skin samples of three antagomiR-203 and three scramble control-injected mice, demonstrated an increase in miR-203 targets Ran, Raph1, Lasp1 and p63 (Figure 2d, left panel). No modulation in miR-203 targets' expression was observed in ventral skin samples from the same mice 
demonstrating the specific and local effect of antagomiR-203 injection (Figure 2d, right panel).

Mir-203 targets control primary keratinocytes proliferation and migration. MiR-203 was described as a 'stemness' repressor and an indirect promoter of differentiation process in epidermal keratinocytes, because of its ability to stop proliferation and block cell cycle at the G0/G1 phase. A leading role in these pathways is probably played by $\Delta \mathrm{Np} 63 \alpha$, which was identified as an miR-203 target. $^{22,26}$ Wound healing requires that keratinocytes at the wound margin increase their proliferative and migratory potential to allow re-epithelialization of the injured area. To evaluate the proliferative and migratory effects of the newly found miR203 target genes, $R A N$ and $R A P H 1$, we employed RNA interference to silence their expression in HEKn cells. To discriminate the specific contribution of different miR-203 targets, we also performed p63 and LASP1 siRNA-mediated silencing, as well as miR-203 overexpression by pre-miR-203 transfection. MiR-203 overexpression resulted in a decrease in cell proliferation as demonstrated by a pulsed 5-bromo-2'deoxyuridine (BrdU) incorporation assay. Cytofluorimetric quantification of BrdU-positive cells as a percentage of the total population was $43 \%$ for scrambled control transfections and $20 \%$ for miR-203 (Figures $3 a$ and b). Propidium iodide (PI) staining of DNA content $48 \mathrm{~h}$ after miR203 transfection showed a G0/G1 population of $66 \%$ compared with $43 \%$ obtained in control cells. Comparable results were obtained both by RAN (19\% BrdU-positive cells, $61 \%$ G0/G1-phase population) and p63 (14\% BrdU-positive cells, 69\% G0/G1-phase population) after siRNA transfection. On the other hand, RAPH1 (43\% BrdU-positive cells, 43\% G0/G1-phase population) and LASP1 (43\% BrdUpositive cells, 42\% G0/G1-phase population) silencing did not affect a cell cycle arrest (Figures $3 a$ and $b$ ). The cell cycle arrest observed after miR-203, RAN and p63 siRNA transfections was never associated with the induction of apoptosis (data not shown).

An evaluation of the migration ability of HEKn cells, silenced as described before, was performed $48 \mathrm{~h}$ after transfection by scratching a confluent cell monolayer in the presence of mitomycin $C$ in an in vitro wound assay. Healing of the scratched area was monitored for $24 \mathrm{~h}$ as illustrated by phasecontrast images in Figure $4 \mathrm{a}$. The histogram in Figure $4 \mathrm{~b}$ quantitatively demonstrates the scratched area's closure by keratinocytes transfected with pre-miR-203 (26\% closure) or a scrambled sequence (68\% closure).

Specific silencing of single miR-203 targets also decreased the migratory ability of primary keratinocytes $(17 \%$ closure for RAN; $55 \%$ for RAPH1; 24\% for p63; 31\% for LASP 1 ), suggesting that all miR-203 targets considered could be involved in wound healing re-epithelialization in vivo. Western blot analysis (Figure 4c) is shown to demonstrate the effective silencing of miR-203 targets obtained by siRNA transfections.

To exclude the possibility that miR-203, through repressing p63, was reducing RAN and RAPH1 expression, we checked their levels in HEKn cells $48 \mathrm{~h}$ after p63 silencing by siRNA. Western blots in Supplementary Figure S4 show that Ran and Raph1 protein levels are not influenced by p63 absence, confirming again that they are direct miR-203 targets.

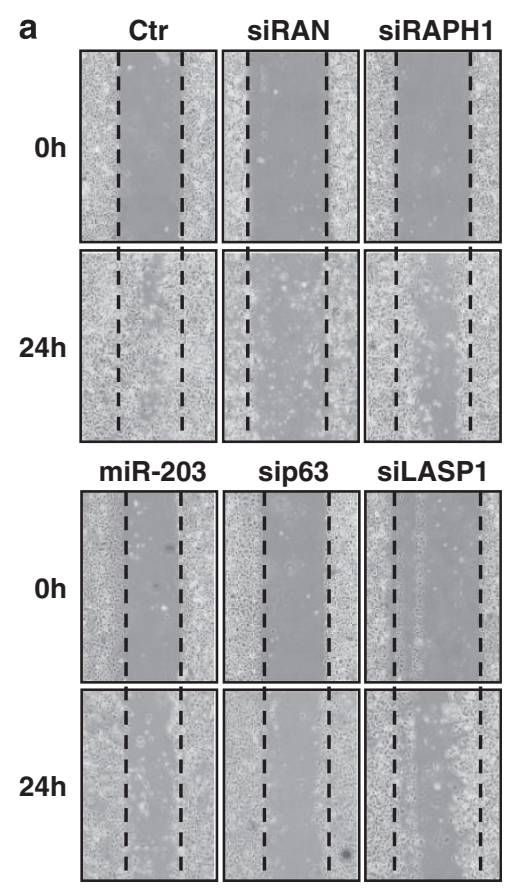

b

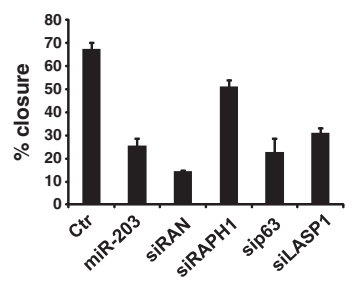

c
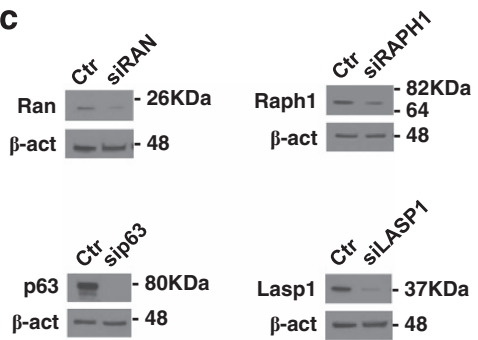

Figure 4 Silencing by siRNA of miR-203 targets reduces the migratory potential of HEKn. (a) In vitro wound assay of HEKn cells, $48 \mathrm{~h}$ after transfection with scramble control (Ctr) or pre-miR-203 sequences. The rate of cell migration across the scratched area was monitored for $24 \mathrm{~h}$ and phase-contrast images are shown. (b) Histogram represents the relative percentage of distance covered by migration $24 \mathrm{~h}$ after scratch (\% closure). Values reported were obtained as the average \pm S.D. of three different measures from three independent assays. (c) Western blot analysis of miR-203 target protein levels in HEKn cells at $48 \mathrm{~h}$ post-transfection with scramble control or target-specific siRNA. $\beta$-Actin was used as a loading control

Characterization of $\mathrm{miR}-203$ target expression in wound healing. Our results indicate that miR-203 targets are proteins expressed in proliferating keratinocytes, necessary for cell migration and, in the case of Ran and p63, also fundamental for cell cycle progression. To analyze, Ran, Raph1, Lasp1 and p63 expression patterns during wound healing, immunohistochemical staining was performed on lesional mouse skin sections collected 3 days post wounding. As shown in Figure 5a, p63-positive cells are 

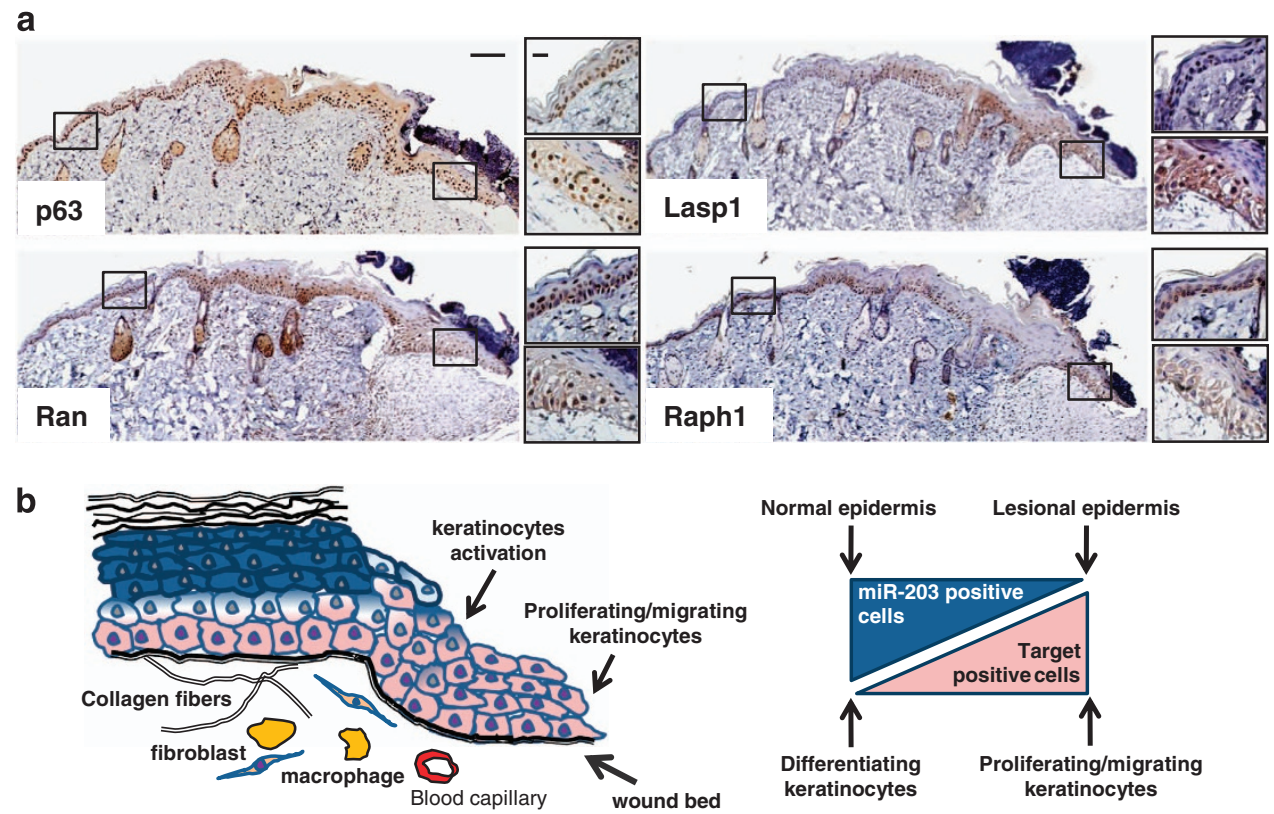

Figure 5 MiR-203 target expression during wound healing. (a) Histological analysis of p63, LASP1, RAN and RAPH1 expression at day 3 after injury. Black bar: $100 \mu \mathrm{m}$. Each panel is a composition of six images taken with an $\times 10$ objective to provide high resolution over a wide area, whereas the insets are a magnification $(\times 20)$ of the identified squared boxes: left box, upper inset; right box, lower inset. Black bar in the insets: $20 \mu \mathrm{m}$. (b) Cartoon model that summarizes the role of miR-203 and its targets during wound healing. Left panel shows a simplified representation of the wound area, where blue cells indicate differentiated keratinocytes and pink cells proliferating keratinocytes. Right panel shows the inverse correlation between the expression of miR-203 (blue) and its targets (pink)

detectable in the basal layer of the normal epidermis. In contrast, a basal and suprabasal localization of p63-expressing cells is evident in the hyperplastic epidermis at the wound margin and in the leading edge of the epithelial tongue. A similar pattern of expression also characterizes Ran, Raph1 and Lasp1 (Figure 5a), resembling a 'photo negative' of miR-203 patterning at the same time post wounding (see In situ hybridization in Figure 1a).

\section{Discussion}

In this study, we have characterized the expression patterns of miR-203 and its targets p63, Lasp1, Ran and Raph1 in mouse skin after wound healing. Recent studies have implicated miRNAs as protagonists in the different phases of wound healing, underlying their role in early inflammation and angiogenesis, but little is known about miRNA expression during later proliferative and remodeling processes. ${ }^{16,55}$ MiR-203 is one of the most abundant skin miRNAs expressed in the suprabasal layers of the mouse epidermis. ${ }^{26}$ Here we have shown for the first time that it is absent in proliferating and migrating keratinocytes at the edge of a wound, while being heavily detectable in the wound's surrounding areas where keratinocytes start to differentiate again to restore a normal stratified epithelium. To better understand this, we made an effort to individuate miR-203 targets possibly implicated in the re-epithelialization pathways. In particular, we identified the GTPase Ran, a proproliferative factor, ${ }^{48-50}$ and Raph1, a promigratory factor, ${ }^{51-53}$ as new putative miR-203 targets. We have demonstrated that they are direct miR-203 targets in vitro and in vivo, in addition to p63 and Lasp1 considered as positive controls. Re-introduction of miR-203 by transient transfection, as well as silencing by siRNA of targets p63 and RAN, reduced the percentage of human primary keratinocytes in the $S$ phase of the cell cycle, while increasing the percentage of those in the G0/G1 phase. Silencing of all miR203 targets considered here impeded the migratory ability of HEKn cells as evaluated by in vitro wound assays. These evidences, together with the coherent expression patterns of miR-203 and its targets in the process of wound healing, suggest that miR-203 repression could mediate the switch to keratinocyte activation during wound closure, whereas its upregulation furnishes a commitment to differentiation both in the healthy and injured epidermis, as proposed in the model described in Figure 5b. Moreover, our results suggest that Ran, Raph1, Lasp1 and p63 play a role in cutaneous wound healing and little is known also about p63 and its targets genes' behavior during re-epithelialization. ${ }^{56,57} \mathrm{MiR}$ 203-mediated control of their expression could be fundamental for skin homeostasis and re-epithelialization. This study warrants further investigations in vivo into the role of these molecular dynamics.

\section{Materials and Methods}

Cell culture and transfection. Human primary epidermal keratinocytes from neonatal foreskin, HEKn (Invitrogen, Carlsbad, CA, USA), were grown in Epilife medium, supplemented with human keratinocyte growth supplement (Invitrogen). HEKn were transfected with human pre-miR-203 and scramble negative control sequences (Ambion, Foster City, CA, USA) using Lipofectamine RNAiMax (Invitrogen) according to the manufacturer's protocol. Silencing of miR203 targets by siRNA was performed using ON-Target plus SMARTpool siRNA (Dharmacon, Lafayette, CO, USA).

Mouse primary keratinocytes were isolated according to Yuspa et al..$^{58}$ from newborn CD1 mice skin. Primary human and mouse keratinocytes were seeded on 
collagen-coated dishes and induced to differentiate by adding $1.2 \mathrm{mM} \mathrm{CaCl}_{2}$ to the culture medium. HEK293E cells were grown in DMEM medium (Invitrogen) supplemented with $10 \%$ fetal bovine serum (Invitrogen) and 1\% penicillin/ streptomycin (Invitrogen).

Mouse tissue collection and antagomiR-203 injection. After being anesthetized, 8-week-old CD1 wild-type mice were shaved and a 4-mm-diameter full-thickness wound was performed on the dorsal midline using a biopsy punch. The wound area was not covered with surgical tape or any medications. At 3 and 5 days after injured mice were killed, the wound site and the surrounding unwounded skin were surgically removed. Specimens were then fixed in $4 \%$ buffered formalin and paraffin embedded. ${ }^{59}$

AntagomiR-203 and scramble control were designed and synthesized (Dharmacon) as described previously. ${ }^{54}$ The antagomiR-203 sequence was $5^{\prime}$ UpsCpsUAGUGGUCCUAAACAUUpsUpsCpsAps-Chol-3' and scramble control sequence was $5^{\prime}$-UpsCpsACAACCUCCUAGAAAGAGUpsApsGpsAps-Chol-3'. Subcutaneous injections were performed on newborn CD-1 mice at a dosage of $80 \mathrm{mg} / \mathrm{kg}$, every day for 3 days. Dorsal and ventral skin samples were collected and used for total RNA and protein extraction.

RNA extraction and quantitative real-time RT-PCR. MirVana miRNA isolation kit (Ambion) was used for total RNA extraction. Relative expression levels of miR-203 were measured, compared with human snU18 as an internal control, by a two-step TaqMan assay according to the manufacturer's instructions (Applied Biosistems, Austin, TX, USA). ${ }^{46}$

In all, $500 \mathrm{ng}$ of total RNA were used for reverse transcription using the InPromll Reverse Transcription System (Promega, Madison, WI, USA), while real-time PCR was performed using Platinum SYBR Green qPCR SuperMix UDG (Invitrogen). Human $\beta$-actin mRNA was used as a housekeeping gene for normalization. ${ }^{46}$ Primer pairs used in PCR reactions are reported in Supplementary Table S1.

Western blotting. Protein extracts and western blots were performed as described before. ${ }^{23}$ The antibodies used were anti-LASP1 (1/10000 dilution; Millipore, Temecula, CA,USA), anti-RAPH1 (1/250 dilution; Sigma, St. Louis, MO, USA), anti-RAN (Sigma; 1/5000 dilution), anti-p63 (1/500 dilution; NeoMarkers, Fremont, CA, USA) and anti- $\beta$-actin (1/5000 dilution; Sigma).

Cell cycle, apoptosis analysis and proliferation assay. For cell cycle analysis, $48 \mathrm{~h}$ post-transfection, cells were harvested as described before. ${ }^{46}$ A total of 10000 events were evaluated using the Cell Quest program (Becton Dickinson, Franklin Lakes, NJ, USA), excluding doublets and aggregates by electronic gating. For proliferation assays, $48 \mathrm{~h}$ post-transfection, Click-iT EdU kit (Invitrogen) was performed according to the manufacturer's instructions.

In vitro migration assay. Cells were transfected and grown to confluency in standard culture medium and conditions. At $48 \mathrm{~h}$ post-transfection, cells were incubated for $2 \mathrm{~h}$ with mitomycin $\mathrm{C}(10 \mu \mathrm{g} / \mathrm{ml})$ to prevent migration by cellular proliferation. In vitro migration assay was then performed as describe before. ${ }^{46}$

Luc assay and constructs. $3^{\prime}$-UTR of miR-203 target mRNAs were amplified by PCR from human genomic DNA using the primer pairs reported in Supplementary Table S2. PCR fragments were restricted and ligated to a compatible Xbal-linearized pGL3Control vector (Promega). Mutagenesis by deletion of miR-203 target sites and luciferase assay were then performed as described before. ${ }^{22}$ Primers pairs are reported in Supplementary Table S2.

Immunohistochemical staining. Morphological analysis of tissue samples was performed by H\&E staining as described previously. ${ }^{60}$ Immunostaining of epithelial markers and miR-203 targets was performed on days 3 and 5 after wounding on paraffin-embedded sections. Briefly, sections were deparaffinized by BioClear (Bio-Optica) and rehydrated. To inactivate endogenous peroxidases, sections were incubated in $3 \% \mathrm{H}_{2} \mathrm{O}_{2}$ in PBS for 15 min. Blocking was carried out in $5 \%$ goat serum in PBS for $1 \mathrm{~h}$ at room temperature. Primary antibody incubation was performed at room temperature for $1 \mathrm{~h}$ : anti-p63 (1/500 dilution; NeoMarkers), anti-K6 (1/800 dilution; Covance, Princeton, NJ, USA), anti-RAN (1/200 dilution; Sigma), anti-RAPH1 (1:100 dilution; Sigma), anti-LASP1 (1/1000 dilution; Millipore). Sections were rinsed in PBS and incubated with DAKO EnVision + System-HRP-labeled polymer (DAKO, Carpinteria, CA, USA). The immune complexes were visualized using $3,3^{\prime}$-diaminobenzidine tetrahydrochloride
(DAKO). Finally, sections were counter-stained with Mayer's hematoxylin (BioOptica) and mounted using EUKITT mounting medium (Bio-Optica). Images of areas of interest were collected by a NIKON Eclipse TE200 microscope equipped with NIKON DS Fi1 camera (Nikon, Tokyo, Japan).

MiRNA in situ hybridization analysis. Formalin-fixed paraffinembedded tissue sections $(6 \mu \mathrm{m})$ were deparaffinized and rehydrated. After treatment with proteinase- $\mathrm{K}\left(2 \mu \mathrm{g} / \mathrm{ml}, 30 \mathrm{~min}, 37^{\circ} \mathrm{C}\right)$, sections were hybridized overnight at $46^{\circ} \mathrm{C}$ with $80 \mathrm{nM}$ miRCURY LNA detection $5^{\prime}$-DIG-labeled probes for U6snRNA, scramble control and miR-203 sequences (Exiqon, Vedbaek, Denmark). After hybridization, sections were washed at the same temperature once in $5 \times$ SSC for $5 \mathrm{~min}$, two times in $1 \times$ SSC for $5 \mathrm{~min}$, three times in $0.2 \times \mathrm{SSC}$ for $5 \mathrm{~min}$ and finally at RT in PBS for $5 \mathrm{~min}$. Blocking was performed in $10 \%$ goat serum in PBS at RT for $1 \mathrm{~h}$, and then sections were incubated for $3 \mathrm{~h}$ in AP-conjugated anti-DIG Fab fragment (1/1500 dilution in blocking buffer; Roche, Mannheim, Germany) at RT. After two washes in PBS for 5 min, detection was performed by incubating in developing buffer (2 mM Levamisole, 1-STEP NBT/ $\mathrm{BCIP}$; Roche) at RT in the dark for $16 \mathrm{~h}$. The reaction was stopped by two washes in Tris- $\mathrm{HCl}(50 \mathrm{mM}), \mathrm{NaCl}(150 \mathrm{mM})$ and $\mathrm{KCl}(10 \mathrm{mM})$ buffer saline. Sections were dehydrated and mounted using EUKITT mounting medium (Bio-Optica, Milano, Italy). Images of the areas were collected as described previously.

Bioinformatics. MiR-203 target sites on the $3^{\prime}$-UTR of putative target genes were predicted by TargetScan 5.1 software available at www.targetscan.org.

\section{Conflict of Interest}

The authors declare no conflict of interest.

Acknowledgements. This work has been supported by 'Alleanza contro il Cancro' (ACC12), MIUR/PRIN (20078P7T3K_001)/FIRB (RBIP06LCA9_0023, RBIP06LCA9_0C), Italian Human ProteomeNet RBRN07BMCT, MIUR/PRIN 2008MRLSNZ_004, Telethon Grant GGP09133 to GM, and by Salute (Ricerca oncologica 26/07) and IDI-IRCCS (RF06 c.73, RF07 c.57, RF08 c.15, RF07 c.57) to GM and EC. The research described in this article was also supported in part by Min.

1. Singer AJ, Clark RA. Cutaneous wound healing. N Engl J Med 1999; 341: 738-746.

2. Grinnel F. Wound repair, keratinocyte activation and integrin modulation. J Cell Sci 1992; 101: 1-5.

3. Coulombe PA. Towards a molecular definition of keratinocyte activation after acute injury to stratified epithelia. Biochem Biophys Res Commun 1997; 236: 231-238.

4. Freedberg IM, Tomic-Canic M, Komine M, Blumenberg M. Keratins and the keratinocyte activation cycle. J Invest Dermatol 2001; 116: 633-640.

5. Santoro MM, Gaudino G. Cellular and molecular facets of keratinocytes reepithelialization during wound healing. Exp Cell Res 2005; 31: 674-686.

6. Proksch E, Brandner JM, Jensen JM. The skin: an indispensable barrier. Exp Dermatol 2008; 17: 1063-1072.

7. Candi E, Schmidt R, Melino G. The cornified envelope: a model of cell death in the skin. Nat Rev Mol Cell Biol 2005; 6: 328-340.

8. Patel GK, Wilson CH, Harding KG, Finlay AY, Bowden PE. Numerous keratinocytes subtypes involved in wound re-epithelialization. J Invest Dermatol 2006; 126: 497-502.

9. Levy V, Lindon C, Harfe BD, Morgan BA. Distinct stem cell populations regenerate the follicle and interfollicular epidermis. Dev Cell 2005; 9: 855-861.

10. Langton AK, Herrick SE, Headon DJ. An extended epidermal response heals cutaneous wounds in the absence of a hair follicle stem cell contribution. J Invest Dermatol 2008; 128: 1311-1318.

11. Paladini RD, Takahashi K, Bravo NS, Coulombe PA. Onset of re-epithelialization after skin injury correlates with reorganization of keratin filaments in wound edge keratinocytes: defining a potential role for keratin 16. J Cell Biol 1996; 236: 231-238.

12. DePianto D, Coulombe PA. Intermediate filaments and tissue repair. Exp Cell Res 2004 301: 68-76.

13. Tomic-Canic M, Komine M, Freedberg IM, Blumenberg M. Epidermal signal transduction and transcription factors activation in activated keratinocytes. J Dermatol Sci 1998; 17: 167-181.

14. Schäfer M, Werner S. Transcriptional control of wound repair. Ann Rev Cell Dev Biol 2007; 23: $69-72$.

15. Gurtner GC, Werner S, Barrandon Y, Longaker MT. Wound repair and regeneration. Nature 2008; 453: 314-321.

16. Banerjee J, Chan YC, Sen CK. MicroRNAs in skin and wound healing. Physiol Genomics 2011; 43: 543-556. 
17. Bartel DP. MicroRNAs: genomics, biogenesis, mechanism and function. Cell 2004; 116 281-297.

18. Krol J, Loedige I, Filipowicz W. The widespread regulation of microRNA biogenesis, function and decay. Nat Rev Genet 2010; 11: 597-610.

19. Aberdam D, Candi E, Knight RA, Melino G. miRNAs, 'stemness' and skin. Trends Biochem Sci 2008; 33: 583-591.

20. Sand M, Gambichler T, Sand D, Skrygan M, Altmeyer P, Bechara FG. MicroRNAs and the skin: tiny players in the body's largest organ. J Dermatol Sci 2009; 53: 169-175.

21. Yi R, Fuchs E. MicroRNA-mediated control in the skin. Cell Death Differ 2010; 17: 229-235.

22. Lena AM, Shalom-Feuerstein R, Rivetti di Val Cervo P, Aberdam D, Knight RA, Melino G et al. miR-203 represses 'stemness' by repressing DeltaNp63. Cell Death Differ 2008; 15: $1187-1195$.

23. Lena AM, Mancini M, Rivetti di Val Cervo P, Saintigny G, Mahé C, Melino G et al. MicroRNA-191 triggers keratinocytes senescence by SATB1 and CDK6 downregulation. Biochem Biophys Res Commun 2012; 423: 509-514.

24. Rivetti di Val Cervo P, Lena AM, Nicoloso M, Rossi S, Mancini M, Zhou H et al. P63 microRNA feedback in keratinocyte senescence. Proc Natl Acad Sci USA 2012; 109 $1133-1138$

25. Sonkoly E, Wei T, Janson PC, Sääf A, Lundeberg L, Tengvall-Linder M et al. MicroRNAs: novel regulators involved in the pathogenesis of psoriasis? PLoS One 2007; 2: e610.

26. Yi R, Poy MN, Stoffel M, Fuchs E. A skin microRNA promotes differentiation by repressing 'stemness'. Nature 2008; 452: 225-229.

27. Senoo M, Pinto F, Crum CP, McKeon F. P63 Is essential for the proliferative potential of stem cells in stratified epithelia. Cell 2007; 129: 523-536.

28. Masse I, Barbollat-Boutrand L, Molina M, Berthier-Vergnes O, Joly-Tonetti N, Martin MT et al. Functional interplay between p63 and p53 controls RUNX1 function in the transition from proliferation to differentiation in human keratinocytes. Cell Death Dis 2012; 3: e318.

29. Barton CE, Johnson KN, Mays DM, Boehnke K, Shyr Y, Boukamp P et al. Novel p63 targe genes involved in paracrine signaling and keratinocyte differentiation. Cell Death Dis 2010; 1: e74

30. Straub WE, Weber TA, Schäfer B, Candi E, Durst F, Ou HD et al. The C-terminus of p63 contains multiple regulatory elements with different functions. Cell Death Dis 2010; 1: e5.

31. Borrelli S, Candi E, Hu B, Dolfini D, Ravo M, Grober OM et al. The p63 target HBP1 is required for skin differentiation and stratification. Cell Death Differ 2010; 17: 1896-1907.

32. Talos F, Wolff S, Beyer U, Dobbelstein M, Moll UM. Brdm2 - an aberrant hypomorphic p63 allele. Cell Death Differ 2010; 17: 184-186.

33. Lena AM, Cipollone R, Amelio I, Catani MV, Ramadan S, Browne G et al. Skn-1a/Oct-11 and $\Delta \mathrm{Np} 63 \alpha$ exert antagonizing effects on human keratin expression. Biochem Biophys Res Commun 2010; 401: 568-573.

34. Shalom-Feuerstein R, Lena AM, Zhou H, De La Forest Divonne S, Van Bokhoven H, Cand $\mathrm{E}$ et al. $\Delta \mathrm{Np} 63$ is an ectodermal gatekeeper of epidermal morphogenesis. Cell Death Differ 2011; 18: 887-896.

35. Aberdam D, Mantovani R. A new p63-deficient mouse model or a fresh look at an old one? Cell Death Differ 2009; 16: 1073-1074.

36. Wolff S, Talos F, Palacios G, Beyer U, Dobbelstein M, Moll UM. The alpha/beta carboxyterminal domains of $p 63$ are required for skin and limb development. New insights from the Brdm2 mouse which is not a complete p63 knockout but expresses p63 gamma-like proteins. Cell Death Differ 2009; 16: 1108-1117.

37. Vanbokhoven H, Melino G, Candi E, Declercq W. P63, a story of mice and men. J Invest Dermatol 2011; 131: 1196-1207.

38. Leonard MK, Kommagani R, Payal V, Mayo LD, Shamma HN, Kadakia MP. $\Delta$ Np63o regulates keratinocyte proliferation by controlling PTEN expression and localization. Cell Death Differ 2011; 18: 1924-1933.

39. Collavin L, Lunardi A, Del Sal G. P53-family proteins and their regulators: hubs and spokes in tumor suppression. Cell Death Differ 2010; 17: 901-911.

40. Browne G, Cipollone R, Lena AM, Serra V, Zhou H, van Bokhoven $\mathrm{H}$ et al. Differential altered stability and transcriptional activity of $\triangle \mathrm{Np} 63$ mutants in distinct ectodermal dysplasias. J Cell Sci 2011; 124(Part 13): 2200-2207.

41. Melino G. P63 is a suppressor of tumorigenesis and metastasis interacting with mutant p53. Cell Death Differ 2011; 18: 1487-1499.
42. Bueno MJ, Pérez de Castro I, Gómez de Cedrón M, Santos J, Calin GA, Ciqudosa JC et al. Genetic and epigenetic silencing of microRNA-203 enhances ABL1 and BCR-ABL1 oncogene expression. Cancer Cell 2008; 13: 496-506.

43. Feber $\mathrm{A}, \mathrm{Xi} \mathrm{L}$, Luketich JD, Pennathur $\mathrm{A}$, Landreneau $\mathrm{RJ}, \mathrm{Wu} \mathrm{M}$ et al. MicroRNA expression profiles of esophageal cancer. J Thorac Cardiovasc Surg 2008; 135: 255-260.

44. Furuta M, Kozaki KI, Tanaka S, Arii S, Imoto I, Inazawa J. MiR-124 and miR-203 are epigenetically silenced tumor-suppressive microRNAs in hepatocellular carcinoma. Carcinogenesis 2010; 31: 766-776.

45. Saini S, Majid S, Yamamura S, Tabatabai L, Suh SO, Shahryari V et al. Regulatory role of miR-203 in prostate cancer progression and metastasis. Clin Cancer Res 2011; 17: 5287-5298.

46. Viticchiè G, Lena AM, Latina A, Formosa A, Gregersen LH, Lund AH et al. MiR-203 controls proliferation, migration and invasive potential of prostate cancer cell lines. Cell Cycle 2011; 10: 1121-1131.

47. Wellner U, Schubert J, Burk UC, Schmalhofer O, Zhu F, Sonntag A et al. The EMT-activator ZEB1 promotes tumorigenicity by repressing stemness-inhibiting microRNAs. Nat Cell Biol 2009: 11: 1487-1495.

48. Rensen WM, Mangiacasale R, Ciciarello M, Lavia P. The GTPase Ran: regulation of cell life and potential roles in cell transformation. Front Biosci 2008; 13: 4097-4121.

49. Yudin D, Fainzilber M. Ran on tracks-cytoplasmic roles for a nuclear regulator. J Cell Sci 2009; 122: 587-593.

50. Jeon H, Zheng LT, Lee S, Lee WH, Park N, Park JY et al. Comparative analysis of the role of small $\mathrm{G}$ proteins in cell migration and cell death: cytoprotective and promigratory effects of RalA. Exp Cell Res 2011; 317: 2007-2018.

51. Lafuente EM, van Puijenbroek A, Krause M, Carman CV, Freeman GJ, Berezovskaya A et al. RIAM, an Ena/VASP and Profilin ligand, interacts with Rap1-GTP and mediates Rap1-induced adhesion. Dev Cell 2004; 7: 585-595.

52. Bae YH, Ding Z, Das T, Wells A, Gertler F, Roy P. Profilin1 regulates PI(3,4)P2 and lamellipodin accumulation at the leading edge thus influencing motility of MDA-MB-231 cells. Proc Natl Acad Sci USA 2010; 107: 21547-21552.

53. Hernández-Varas P, Coló GP, Bartolomé RA, Paterson A, Medraño-Fernández I, ArellanoSánchez N et al. Rap1-GTP-interacting adaptor molecule (RIAM) protein controls invasion and growth of melanoma cells. J Biol Chem 2011; 286: 18492-18504.

54. Krützfeldt J, Rajewsky N, Braich R, Rajeev KG, Tuschl T, Manoharan M et al. Silencing of microRNAs in vivo with 'antagomirs'. Nature 2005; 438: 685-689.

55. Shilo S, Roy S, Khanna S. MicroRNA in cutaneous wound healing: a new paradigm. DNA Cell Biol 2007; 26: 227-237.

56. Bamberger $\mathrm{C}$, Hafner A, Schmale H, Werner S. Expression of different p63 variants in healing skin wounds suggests a role of $p 63$ in reepithelialization and muscle repair. Wound Repair Regen 2005; 13: 41-50.

57. Koster MI, Dai D, Marinari B, Sano Y, Costanzo A, Karin M et al. P63 induces key target genes required for epidermal morphogenesis. Proc Natl Acad Sci USA 2007; 104: 3255-3260.

58. Yuspa SH, Kilkenny AE, Steinert PM, Roop DR. Expression of murine epidermal differentiation markers is tightly regulated by restricted extracellular calcium concentrations in vitro. J Cell Biol 1989; 109: 1207-1217.

59. Cianfarani F, Zambruno G, Brogelli L, Sera F, Lacal PM, Pesce M et al. Placenta growth factor in diabetic wound healing. Altered expression and therapeutic potential. Am J Pathol 2006; 169: 1167-1182.

60. Candi E, Rufini A, Terrinoni A, Giamboi-Miraglia A, Lena AM, Mantovani R et al. DeltaNp63 regulates thymic development through enhanced expression of FgfR2 and Jag2. Proc Nat Acad Sci USA 2007; 104: 11999-12004.

Cell Death and Disease is an open-access journal published by Nature Publishing Group. This work is licensed under the Creative Commons Attribution-NonCommercial-No Derivative Works 3.0 Unported License. To view a copy of this license, visit http://creativecommons.org/licenses/by-nc-nd/3.0/ 